\title{
A chacun ses valeurs
}

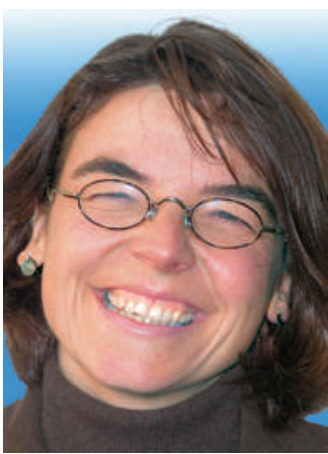

Christina Aus der Au

A lire également à ce sujet, l'article à la page 929.
La provocation est un moyen tout à fait légitime (et qui plus est souvent jouissif) de susciter une prise de conscience de l'interlocuteur. Je suis donc très reconnaissante à Messieurs Menuz et Roduit d'avoir attiré mon attention sur l'article signé M. Liao, A. Sandberg et R. Roache. Et, grâce à Internet, sur les débats passionnés qu'il a généré dans les courriers de lecteurs et blogs anglo-saxons. Cette polémique m'a surtout confortée dans la conviction qu'aucun sujet n'était interdit. On doit aussi pouvoir énoncer presque tout, avec toute la prudence nécessaire. Peut-être existe-t-il quelques exceptions, mais selon moi, réfléchir publiquement et sans aucun tabou à la façon d'aborder le problème du réchauffement climatique n'en est pas une.

Même si l'on envisage pour cela de toucher à l'être humain. La moralisation, le recours aux nouvelles technologies et aux marchés durables et l'action à grande échelle au niveau du cycle de $\mathrm{CO}_{2}$ feraient place à une intervention ciblée sur l'auteur du problème, à savoir l'être humain. C'est en fait une méthode déjà pratiquée dans de nombreux domaines avec beaucoup de succès, consistant à prendre le mal à la racine, selon le principe de causalité. En d'autres mots, s'attaquer à cette insatiable faim de viande, à l'expansion croissante de l'espèce et de l'individu en particulier, enfin à l'égoïsme des hommes et au manque d'empathie.

\section{«Qui définirait les besoins? A qui profiterait cette évolution?»}

\footnotetext{
* Christina Aus der Au, docteur en théologie, privat-docent en théologie systématique/ dogmatique de l'université de Bâle et membre de la rédaction Ethique du Bulletin des médecins suisses
}

Influencer tous ces facteurs à peu de frais et à grande échelle, rendre les hommes moins nombreux et moins cupides et faire en sorte qu'ils mangent moins, qu'est-ce qui s'oppose à cette manière de réduire le $\mathrm{CO}_{2}$ ?

Sinon l'horrifiante pensée qu'on interviendrait là de la façon la plus sinistre sur l'essence même de l'homme. Que les droits de l'individu à la liberté seraient foulés aux pieds sur l'autel du bien de la communauté. Que c'est l'âme de l'homme et non son entité physique qui serait altérée. Et qui définirait les besoins? A qui profiterait cette évolution?

Le côté passionnant de cette expérience intellectuelle (que j'avoue ne pas vouloir pousser plus loin pour l'instant) est de révéler les enjeux réels. D’un côté la menace d'une catastrophe climatique, avec la sécheresse, les tempêtes, les inondations, la perte des terres et des maisons, les maladies et les immenses coûts que cela entraîne. De l'autre, les valeurs telles que la libre disposition de soi-même, l'individualité, les droits de l'homme, la démocratie, l'intégrité personnelle, la justice et la liberté.

On pourrait penser qu'il s'agit tout bonnement de choisir entre l'utilitarisme et le plus grand profit pour le plus grand nombre possible d'une part et une éthique du devoir, avec des normes et valeurs non négociables d'autre part.

Mais qui défend ces principes éclairés et libéraux? N'est-ce pas nous, Américains et Européens continentaux, qui sommes bien assurés et protégés, qui ne vivons pas de nos terres mais importons nos denrées du monde entier, et dont les maisons ne sont pas englouties par la mer ni arrachées par les ouragans. Alors qu'en face, ceux qui risquent de perdre leurs moyens de subsistance se moquent, dans une telle situation, de sacrifier $10 \mathrm{~cm}$ de leur taille, une constitution génétique et hormonale «naturelle», ou le goût de la viande, qu'ils ne peuvent de toute façon probablement pas s'offrir.

Sur cette question, rester attachés à nos belles valeurs et à nos principes ne relève-t-il pas d'un luxe que nous ne pouvons nous offrir que parce que nous ne sommes pas autant touchés que les habitants des pays insulaires et des nations agricoles pauvres? Ne serait-il pas grand temps que nous réfléchissions à notre participation à la réduction de $\mathrm{CO}_{2}$ ? A savoir, puisque cela ne se fait manifestement pas de façon volontaire, comme en témoignent les conventions, mandats et protocoles depuis Rio en 1992, rendre au moins cette participation obligatoirement volontaire, en acceptant les interventions génétiques et hormonales sur les êtres humains récalcitrants que nous sommes, pour le bien de l'humanité.

On pourrait argumenter de cette façon au niveau éthique, à juste titre. Pourtant, une voix persiste à me dire que ces interventions sur l'être humain nous feraient perdre quelque chose de grand et de profond. A savoir, relever le défi de dépasser le stade d'Homo faber, dans un monde manipulable à merci, pour être un Homo sapiens capable d'écouter, de s'étonner et d'aimer, qui non seulement aurait de nombreuses connaissances mais saurait en plus les utiliser avec sagesse.

Christina Aus der Au* 